\title{
Upper mediastinal and paratracheal node dissection in total (pharyngo) laryngectomy, it is really indicated?
}

\author{
Didier Dequanter, M. Shahla, P. Paulus, P. Lothaire
}

Department of Head and Neck Surgery, CHU Charleroi, Montigny le Tilleul, Belgium.

Email: Didier.dequanter@pandora.be

Received 25 July 2011; revised 29 August, 2011; accepted 14 September 2011.

\section{ABSTRACT}

Introduction: Advanced laryngeal and hypopharyngeal cancers are aggressive tumors with a poor prognosis. Multiple lymph node metastases often occur in the neck as well as in the upper mediastinum and thus upper mediastinal dissection is crucial to improving the cure rate. However, excessive mediastinal dissection can increase postoperative morbidity and mortality making it important to employ the proper technique and appropriate extent of dissection. In the present study, we aimed to determine the need and the prognostic importance of mediastinal dissection in patients with advanced carcinoma of the upper aerodigestive tract. Methods: A retrospective review of the records of 30 patients who underwent (pharynxgo) laryngectomy for advanced squamous cell carcinomas was done. 17 patients had laryngeal carcinomas, 13 had hypopharyngeal carcinomas. The mediastinal dissection was designed to remove mainly the paratracheal and retrooesophageal lymph nodes. Results: 60 neck dissections and 30 mediastinal dissection were performed in 30 patients and yielded positive nodes were found in $20 / 30$ patients. Neck nodes were positive in $9 / 17$ of the patients with laryngeal cancer and 11/13 of the patients with hypopharyngeal cancers respectively. Positive nodes were detected in the neck regardless of $T$ stage. The mediastinal nodes were positive in $0 \%$ of the patients with laryngeal cancer. Upper mediastinal metastases were detected positive in 6/13 of the hypopharyngeal patients. In these patients, mediastinal metastases were associated with tumors greater than $35 \mathrm{~mm}$. The majority of positive paratracheal nodes were less than 1 $\mathrm{cm}$ in diameter and appeared negative preoperatively. $0 \%$ of the patients had positive paratracheal nodes alone in a histologically negative cervical neck dissection Regarding the appropriate extent of dissection, no major complications were observed. Conclusions: There is little controversy about neck dissection in advanced tumors of the (pharyngo) larynx. Laryn- geal carcinomas showed no positive mediastinal nodes in this series. The study highlighted the propensity of advanced hypopharyngeal cancers to involve the paratracheal nodes.

Keywords: Advanced Head and Neck Cancer; Mediastinal Dissection; Hypopharynx

\section{INTRODUCTION}

(Pharyngo) laryngeal squamous cell carcinoma is a very aggressive cancer that is generally diagnosed at advanced stages and, consequently, has a poor prognosis and low survival. More than $75 \%$ of patients with (pharyngo) laryngeal tumors have stage III or IV disease at presentation [1]. Indeed, cervical metastases are present in $60 \%-80 \%[2,3]$ and, often, associated with multiple lymph nodes metastases that occur in the upper mediastinum as well as in the paratracheal area. Although neck dissection is the surgical standard for the treatment of cervical lymph spread the upper mediastinal paratracheal nodal group is not routinely included in the dissection. However, metastases to paratracheal lymph nodes occur in $30 \%$ of patients, mostly with hypopharyngeal tumors [4]. A $20 \%$ frequency of occult metastases to ipsilateral paratracheal lymph nodes has been reported in laryngeal cancer patients with postcricoid lesions and in patients with tumors that involved the pyriform fossa [5]. Moreover, laryngeal/hypopharygeal metastatic disease involving the paratracheal nodal group has been implicated in recurrent disease, particulary in patients with peristomal recurrence; this suggest the prognostic significance of positive paratracheal metastatic spread $[6,7]$.

The purpose of this study was to analyse the paratracheal nodal yield and frequency of metastases and determine the indications of the upper mediastinal paratracheal dissection.

\section{METHODS}

The study group consisted of 30 patients that were not previously treated for advanced squamous cell carcinoma 
(SCC) of the (pharyngo) larynx. They underwent surgery from May 2007 to July 2010. The cases were reviewed retrospectively. The diagnostic evaluation at presentation included a complete physical examination, panendoscopy, CT and /or MRI scans of the head and neck, chest $\mathrm{X}$-rays, and laboratory tests. We assessed the CT and/or the RMN to determine the regional lymph node status. No patient had radiological evidence of distant metastasis at presentation, and all patients were treated with curative intent. At our institution in principal the choice of radical therapy is surgery for advanced tumors and neck lymph node metastasis is treated by neck dissection. Additional radio (chemo) therapy in conducted in patients with multiple lymph node metastasis.

To determine the significance between paratracheal lymph node metastasis and clinical factors (such as age, gender, primary tumor site, TN stage, pTN stage, the chi-square test, Fisher's exact test, multiple logistic regression analysis, multiple linear regression analysis, and correlation analysis were used, as appropriate. A p < 0.05 was considered statistically significant.

\section{RESULTS}

The median age was 58 years (range 43 - 90 years); there were 25 males and 5 females the follow-up period ranged from 3 to 41 months with a mean of 27 months.

The site of the original primary tumor was recorded in all patients and included: 17 patients with a laryngeal cancer and 13 patients with hypopharyngeal tumor.

Concerning the disease stage of the cervical lymph nodes, there were $6 \mathrm{~N} 0$ patients; $4 \mathrm{~N} 1$ patient and $20 \mathrm{~N} 2$ patients

Regarding the pathological stage, 14 patients had T3 staged cancers and 16 patients had T4 cancers.

Regarding the cell differentiation, there were 12 welldifferentiated, 9 moderate-differentiated, and 9 poorly differentiated cases, respectively.

For tumor excision, 17 patients had total laryngectomy and 13 pharyngolaryngectomy. Laryngeal resection was performed under direct vision based on the invasion of the tumor. The surgical defects were reconstructed with pectoralis major myocutaneous flap in 13 cases. All the patients had neck dissection at the time of the primary surgery. A modified bilateral radical neck dissection was performed in all cases. All the patients had a bilateral paratracheal lymph node dissection. The paratracheal node dissection included dissection of all of the nodes between the carotid artery laterally and the trachea medially, as far inferior into the superior mediastinum as possible through the cervical approach. There was no preoperative mortality.

The rate of ipsilateral paratracheal lymph nodes metastasis was $6 / 30$. No patients had controlateral paratracheal nodes.
The mean number of paratracheal lymph nodes dissected per side was 2.3 .

Histological paratracheal metastasis in the clinically node positive neck were present in patients with pyriform sinus cancer. All the patients had a tumor greater than $35 \mathrm{~mm}$. In the clinically node negative, no paratracheal histological metastases were found.

At the end of the follow-up, 10/30 died. More specifically, $3 / 6(50 \%)$ of the patients with positive paratracheal nodes died.

We analysed the correlation between paratracheal lymph nodes metastasis and several clinical factors among the 30 patients (Table $\mathbf{1}$ ).

There was a strong but not statistically significant correlation of paratracheal lymph node metastasis with the primary site. However, there was no significant relationship between age, gender, TN stage and pTN stage and paratracheal lymph node metastasis.

\section{DISCUSSION}

Advanced laryngeal and hypopharyngeal carcinomas had a high tendency to invade cervical lymph nodes. This nodal extension is a well-known prognostic factor [7$10]$.

Table 1. Clinical factors affecting paratracheal lymph node metastasis in patients with laryngeal and hypopharyngeal carcinomas.

\begin{tabular}{|c|c|c|c|}
\hline Parameters & $\begin{array}{l}\text { PTN positive } \\
\quad(n=6)\end{array}$ & $\begin{array}{l}\text { PTN negative } \\
\quad(\mathrm{n}=24)\end{array}$ & $P$ value \\
\hline $\begin{array}{l}\text { Age } \\
<60 \\
\geq 60\end{array}$ & $\begin{array}{l}3(17 \%) \\
3(25 \%)\end{array}$ & $\begin{array}{c}15(83 \%) \\
9(75 \%)\end{array}$ & 0.57 \\
\hline $\begin{array}{l}\text { Gender } \\
\text { Male } \\
\text { Female }\end{array}$ & $\begin{array}{c}6(24 \%) \\
0(0 \%)\end{array}$ & $\begin{array}{l}19(76 \%) \\
5(100 \%)\end{array}$ & 0.54 \\
\hline $\begin{array}{l}\text { Primary site } \\
\text { Larynx } \\
\text { Hypopharynx }\end{array}$ & $\begin{array}{l}0(0 \%) \\
6(38 \%)\end{array}$ & $\begin{array}{c}16(100 \%) \\
8(62 \%)\end{array}$ & 0.08 \\
\hline $\begin{array}{c}\text { T clinic stage } \\
\text { T3 } \\
\text { T4 }\end{array}$ & $\begin{array}{c}0(0 \%) \\
6(26 \%)\end{array}$ & $\begin{array}{l}7(100 \%) \\
17(74 \%)\end{array}$ & 0.77 \\
\hline $\begin{array}{c}\text { T anapathologica } \\
\text { stage } \\
\text { T3 } \\
\text { T4 }\end{array}$ & $\begin{array}{c}0(0 \%) \\
6(38 \%)\end{array}$ & $\begin{array}{c}14(100 \%) \\
10(62 \%)\end{array}$ & 0.54 \\
\hline $\begin{array}{c}\text { N clinic stage } \\
\text { N0 } \\
\text { N1 } \\
\text { N2 }\end{array}$ & $\begin{array}{l}0(0 \%) \\
0(0 \%) \\
6(30 \%)\end{array}$ & $\begin{array}{l}6(100 \%) \\
4(100 \%) \\
14(70 \%)\end{array}$ & 0.58 \\
\hline $\begin{array}{c}\mathrm{N} \text { anapathologica } \\
\text { stage } \\
\mathrm{N} 0 \\
\mathrm{~N} 1 \\
\mathrm{~N} 2\end{array}$ & $\begin{array}{c}0(0 \%) \\
0(0 \%) \\
6(38 \%)\end{array}$ & $\begin{array}{l}9(100 \%) \\
4(100 \%) \\
10(62 \%)\end{array}$ & 0.36 \\
\hline
\end{tabular}


Although neck dissection is the surgical gold standard for the treatment of cervical lymphatic spread, the upper mediastinal and paratracheal nodal groups are not routinely included in the dissection.

However, Welsh et al. [11] initially reported the importance of the paratracheal nodes in lymphatic drainage of the larynx and hypopharynx. Harrison et al. confirmed that carcinomas of the larynx (especially from the subglottic region), the trachea, and the cervical esophagus are at risk for paratracheal and tracheobronchial lymph node metastasis and recommended resection of the manubrium to remove theses lymph nodes and reduce the risk for parastomal disease recurrence [5]. Weber et al. [7] reported that paratracheal lymph node metastasis were found in $17.6 \%$ of the patients with tumors of the larynx, in $8.3 \%$ of the patients with pharyngeal tumors and, at least, in $71.4 \%$ of the patients with tumors of the esophagus.

Some investigators have suggested that ipsilateral paratracheal node dissection should be included as part of a selective neck dissection in all patients with tumors invading the subglottis, pyriform fossa apex and postcricoid region [12].

In our study, all the patients with advanced laryngeal and hypopharyngeal SCC had a neck dissection extended to the upper mediastinum. Most patients with hypopharyngeal SCC had positive paratracheal lymph nodes but only to the ipsilateral side of the primary. All the patients with hypopharyngeal SCC greater than $35 \mathrm{~mm}$ had paratracheal lymph node metastasis. Furthemore, paratracheal lymph node metastasis was always associated with the presence of cervical lymph node metastasis. In addition, a strong correlation but, unfortunately, not statistically significant was found between the presence of paratracheal lymph node metastasis and hypoharyngeal tumors.

We recommend that ipsilateral paratracheal node dissection should be included as part of a selective neck dissection in all patients with tumors invading the hypopharynx, certainly in tumors greater than $35 \mathrm{~mm}$.

In terms of survival rates, in a prospective study of 50 patients with carcinoma of the larynx, hypopharynx, and cervical esophagus, Timon et al. [13] reported that the rate of paratracheal nodal metastases was $26 \%$. Moreover, they reported that the survival in patients with positive paratracheal nodes demonstrated a trend towards poorer survival compared to patients without paratracheal nodal involvement, and concomitant involvement of both cervical and paratracheal nodal groups was associated with the poorest survival propability.

In their study, Weber et al. [7] confirmed that survival was significantly reduced by the presence of paratra- cheal lymph nodes. In our study, $50 \%$ of the patients with positive paratracheal nodes died.

In conclusion, there is little controversy about neck dissections in advanced tumors of the larynx and hypopahrynx. A similar situation applies to mediadtinal dissection for hypopharyngeal carcinomas, certainly in tumors greater than $35 \mathrm{~mm}$.

\section{REFERENCES}

[1] Hoffman, H.T., Karnell, L.H., Funk, G.F., Robinson, R.A. and Menck, H.R. (1998) The National Cancer Data Base Report on cancer of the head and neck. Archives of Otolaryngology-Head \& Neck Surgery, 124, 951-962.

[2] Ho, C.M., Lam, K.H., Wei, W.I., Yuen, P.W., Lam, L.K. (1993) Squamous cell carcinoma of the hypopharynxAnalysis of treatment results. Head Neck, 15, 405-412. doi:10.1002/hed.2880150507

[3] Lefebvre, J.L., Castelain, B., DeLatorre, J.C., DelobelleDeroide, A. and Vankemmel, B. (1987) Lymph node invasion in hypopharynx and lateral epilarynx: A prognostic factor. Head \& Neck Surgery, 10, 14-18. doi:10.1002/hed.2890100104

[4] Wei, W.I. (2002) The dilemma of treating hypopharyngeal carcinoma: More or less. Archives of Otolaryngology-Head \& Neck Surgery, 128, 229-232.

[5] Buckley, J.G. and Lemman, K. (2000) Cervical node metastases in laryngeal and hypopharyngeal cancer: A prospective analysis of prevalence and distribution. Head Neck, 22, 280-385.

doi:10.1002/1097-0347(200007)22:4<380::AID-HED11 $>3.0 . \mathrm{CO} ; 2-\mathrm{E}$

[6] Harris, H.H. and Butler, E. (1968) Surgical limits in cancer of the subglottic larynx. Archives of Otolaryngology, 87, 64-67.

[7] Weber, R.S., Marvel, J., Smith, P., Hankins, P., Wolf, P. and Goepfert, H. (1993) Paratracheal lymph node dissection for carcinoma of the larynx, hypopharynx and cervical esophagus. Archives of Otolaryngology-Head \& Neck Surgery, 108, 11-17.

[8] Driscoll, W.G., Nagorsky, M.J., Cantrell, R.W. and Johns, M.E. (1983) Carcinoma of the pyriform sinus: Analysis of 102 cases. Laryngoscope, 93, 556-560.

[9] Bataini, J.P., Bernier, J., Brugere, J., Jaulerry, C., Picco, C. and Brunin, F. (1985) Natural history of neck diseases in patients with squamous cell carcinoma of oropharynx and pharyngolarynx. Radiotherapy \& Oncology, 21, 245255. doi:10.1016/S0167-8140(85)80033-5

[10] Lindberg, R. (1972) Distribution of cervical lymph nodes metastases from squamous cell carcinoma of the upper respiratory and digestive tracts. Cancer, 29, 1446-1449. doi:10.1002/1097-0142(197206)29:6<1446::AID-CNCR 2820290604>3.0.CO;2-C

[11] Welsh, L.W. (1964) The normal human laryngeal lymphatics. Annals of Otology, Rhinology, and Laryngology, 73, 569-582.

[12] Harrison, D.F.N. (1975) Laryngectomy for subglottic lesions. Laryngoscope, 85, 1208-1210. 
doi:10.1288/00005537-197507000-00012

[13] Timon, C.V., Toner, M. and Conlon, B.J. (2003) Paratracheal lymph node involvement in advanced cancer of the larynx, hypopharynx and cervical esophagus. Laryngoscope, 113, 1595-1599.

doi:10.1097/00005537-200309000-00035 